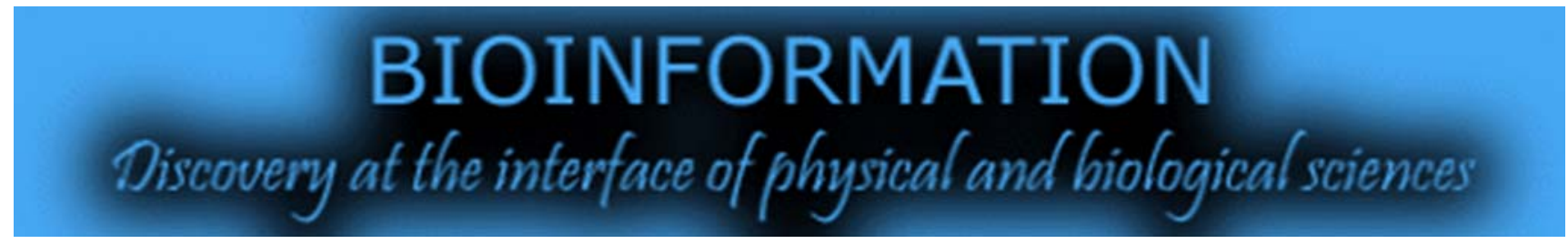

open access

www.bioinformation.net

Current Trends

Volume 7(6)

\title{
Dissemination of Evidence-Based Standards of Care
}

\author{
Andre Barkhordarian', ${ }^{1}$, Brett Hacker2, 3, Francesco Chiappelli1, 2* \\ ${ }^{1}$ Oral Biology \& Medicine, School of Dentistry, UCLA; ${ }^{2}$ Evidence-Based Study Group, Evidence-Based Decisions Practice-Based \\ Research Network (ebd-pbrn.org); ${ }^{3}$ Director, IT Software Development, Live Nation. Francesco Chiappelli - Email: \\ fchiappelli@dentistry.ucla.edu; *Corresponding author
}

Received November 12, 2011; Accepted November 16, 2011; Published November 20, 2011

\begin{abstract}
:
Standards of care pertain to crafting and implementing patient-centered treatment interventions. Standards of care must take into consideration the patient's gender, ethnicity, medical and dental history, insurance coverage (or socioeconomic level, if a private patient), and the timeliness of the targeted scientific evidence. This resolves into a process by which clinical decision-making about the optimal patient-centered treatment relies on the best available research evidence, and all other necessary inputs and factors to provide the best possible treatment. Standards of care must be evidence-based, and not merely based on the evidence - the dichotomy being critical in contemporary health services research and practice. Evidence-based standards of care must rest on the best available evidence that emerges from a concerted hypothesis-driven process of research synthesis and meta-analysis. Health information technology needs to become an every-day reality in health services research and practice to ensure evidence-based standards of care. Current trends indicate that user-friendly methodologies, for the dissemination of evidence-based standards of care, must be developed, tested and distributed. They should include approaches for the quantification and analysis of the textual content of systematic reviews and of their summaries in the form of critical reviews and lay-language summaries.
\end{abstract}

Keywords: evidence-based decision-making, standard of care, systematic reviews, text mining, contextual analysis, quantification

\section{Background:}

Current trends indicate that the dissemination of novel, timely and critical bio-medical information stands as the next frontier of health care. The development of technologies that favor and enable clinical practice guidelines that are based on new and emerging research evidence, is at the cusp of the modernization of public and private health care services, and the task of identifying, packaging and disseminating evidence-based standards of $\operatorname{care}^{1}$ (see supplementary material) lies at the core of expanding health information technologies. Standards of care must be evidence-based, and not based on the evidence - a critical dichotomy in contemporary health services research and practice: they must integrate the best available evidence that emerges from a concerted hypothesis-driven process of research synthesis and meta-analysis [1]. They should implement patient-centered treatment interventions while considering the

ISSN 0973-2063 (online) 0973-8894 (print)

Bioinformation 7(6): 315-319 (2011) patient's gender, ethnicity, health care history, coverage, and socioeconomic level, by integrating the best available patienttargeted scientific evidence.

Patient-centered treatment is optimized through effective dissemination of the best available research evidence, and all other necessary inputs and factors to provide optimal treatment through well-crafted and coordinated health information technologies. Novel approaches for the quantification and analysis of the textual content of systematic reviews and of their summaries in the form of critical reviews and lay-language summaries represent the next frontier of health information technology [1, 2].

Evidence-based clinical decision-making, the process of making diagnostic and prognostic decisions for health care that are 
based on the best available evidence [3], rests on obtaining the best available evidence through the systematic hypothesisdriven process of research synthesis that is framed by the characteristics of the patient population under study, the interventions under consideration, and the clinical outcomes being sought (P.I.C.O.). The P.I.C.O.-driven process uncovers all of the available literature pertinent to the research question the bibliome -, which is evaluated for the level and the quality 2 of the evidence. Analyses of the data ascertain the best quality reports, and yield over-arching evaluations of the effects (i.e., meta-analysis). Reports that describe P.I.C.O.-driven research and analysis, termed systematic reviews, are complex, sophisticated, and are not user-friendly [1-3].

Critical summaries (aka, evidence reviews) of systematic reviews, and lay-language summaries of critical summaries are produced through a stringent writing protocol, and are rigorously evaluated for clinical relevance to disseminate the information in systematic reviews to the clinicians as well as the patients. The American Dental Association (ADA) has stated that $^{3}$ (see supplementary material): “development of a critical summary follows a comprehensive process of critical appraisal, which focuses on assessing the methodological rigor of a published systematic review and the clinical utility and validity of the review methodology, design and evidence analysis. Critical summaries provide a concise, focused summary of a published systematic review: evaluating the appropriateness of the review's search strategy and execution; the review's appraisal of the available evidence and other considerations. Each critical summary includes ratings of the systematic review findings and their clinical applicability and relevance, and addresses how this information may be applied in patient care"

A critical summary, the ADA Center for Evidence-Based Dentistry states, consists of: (a) Critical summary title and author, distinct from the systematic review title and author(s) (b) Overview: (1) Systematic Review Conclusion; (2) Overall Critical Assessment; (3) Level of Evidence Rank (i.e., SORT; [5]). (c) Structured abstract to describe (1) P.I.C.O; (2) bibliome; (3) findings; (4) conclusions; (5) source of funding (to ascertain absence of conflict of interests).(d) Commentary: 300-400 words about (1) clinical context; (2) strengths/weaknesses of the systematic review; (3) strengths/weaknesses of the evidence; (4) implications for evidence-based practice. Critical reviews must be translated in lay language to empower the patients by increasing health literacy, and such lay-language summaries must also be disseminated through health information technology [2, 4, 6]. Moreover (or furthermore), according to ADA Center for Evidence-Based Dentistry: "plain language summaries provide professionals and patients with one page documents that summarize systematic reviews in a consumer friendly manner".

A lay-language summary should consist of the following [4]: (a) Lay-language summary title and author, distinct from the systematic review and the critical summary titles and author. (b) Overview: (1) Systematic review and critical review full citation and link; (2) Clinical questions; (3) Consensus of the best available evidence. (c) Background: (1) Glossary; (2) Background information; (d) Conclusion: Research must develop and validate novel methodologies for ensuring the quality of the communications, and the efficiency of the disseminative process. This is particularly timely and critical in the context of the patient-centered medical/dental home /neighborhood [7], and of the trans-national and global perspective of translational effectiveness and health information technology [2].

From a methodological standpoint, a simple checklist can rank critical reviews and lay-language summaries in terms of whether they are written in a direct, precise and succinct format that contains the main points highlighted above. A measure of quality on continuous or semi-continuous scale will require: (a) Construct validation: conceptualization of the fundamental elements of a critical review and lay-language summary; (b) Content validation: item development and analysis (i.e., factor analysis, cluster analysis) of items targeted to assess the fundamental aspects of the critical review and lay-language summary; (c) Reliability: Internal consistency (i.e., Cronbach $\alpha$ ), inter-rater reliability, and Cohen $\kappa$ coefficient of agreement); (d) Contextual extraction: quantification of the salient qualitative statements about the reported best available evidence by means of text mining and analysis [8]. The latter entails the very essence of the best available evidence, which should permit clinicians to make better informed decisions for treatment, and will empower patients by raising health literacy and overall satisfaction.

The presentation and dissemination of high quality and validated critical reviews and lay-language summaries through user-friendly and easily accessible health information technologies will be an essential turning point in the mode and delivery of health care in the XXI century. In computer language, information is expressed as binary ( 0 or 1$)$ bits. One byte ( 8 bits) represents a digit, a letter or a symbol. A total of 1.8 zettabyte (ZB) digital data was created in $2011(1 \mathrm{ZB}=$ a trillion gigabytes (GB) $\left(1 \mathrm{~GB}=10^{9}\right.$ bytes, $2^{30}$ binary bytes, is about $7 \mathrm{~min}$ of HDTV). From ZB (1021, binary 270 ), the next frontier of information technology is the yottabyte (1024 bytes), and thence beyond. The gargantuan increase in memory capacity of information technology is related to fast accelerated computational speed, from a tenth of a second in the first Electronic Numerical Integrator And Computer (ENIAC, mid1940 's) to the close to 10 quadrillionth of a second of today's most powerful machines KEI computers ${ }^{4}$ (see supplementary material). These increases in performance were predicted as far back as 1965 by Intel co-founder Gordon E. Moore (1929 - ), in that processor speed would double every 12 months. He revised this prediction in 1975, stating that processor speed would double every 24 months. His predictions, now commonly referred to as "Moore's Law", have proven accurate over the years.

Following in this trend, gigantic databases are also being amassed: (a) Natural science databases - (1) National Geographic-initiated project to collect and catalogue DNA profiles across the world to trace human history ("Genographic" project, 2005); (2) "encyclopedia of life" (eol.org project) to track the over 2 million species; (3) "combined DNA index system, CODIS" (FBI, 1998) to store genetic and genomic data for forensic purposes; (4) "FAOSTAT and TERRASTAT" databases of the United Nations to monitor food, land and water supplies worldwide; (5) digitalized survey of the night sky ("Sloan 
Digital Sky Survey, SDSS"), to scrutinize the outer frontiers of the universe in search for extra-terrestrial life. (b) psycho-social databases - (1) "MD: $\mathrm{PRO}^{\prime}$, centralized anti-malicious hardware and software collection center; (2) "OKTRENDS" (OKCupid.com) for analyzing and cataloging human behavior. (c) Information databases; (d) "worldcat" (world.cat.org) to collect the catalogues of over a thousand libraries in 112 countries in the global Online Computer Library Center (OCLC); (e) "Wayback" archival system of the web (archiveaccess.sourceforge.net/projects/wayback, 1996) to provide free access to nearly 3 million public domain written documents.

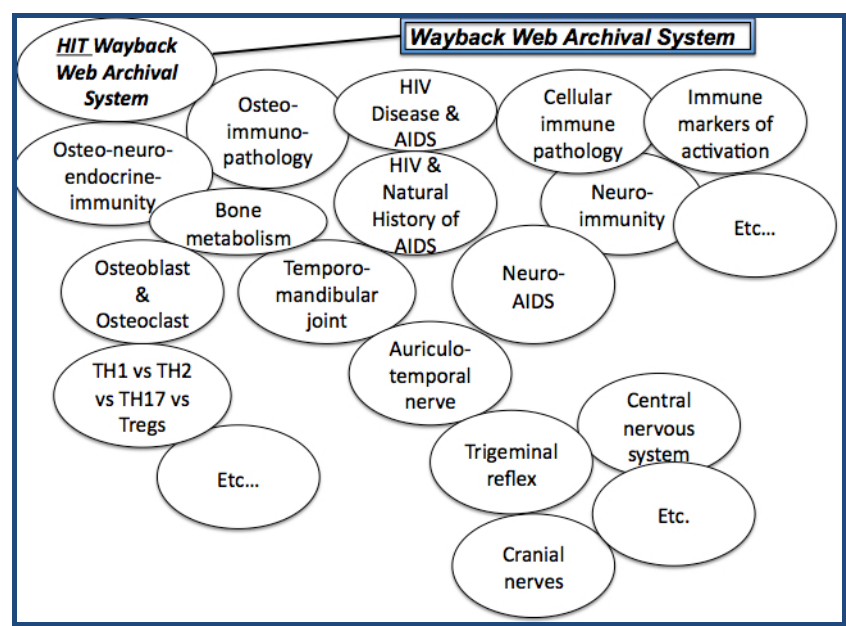

Figure 1: Putative Health Information-Wayback - OsteoImmunopathology. Looking at the future of health information technology, one may conceive of a Health InformationWayback structure that geometrically expands Sir T. BrennersLee's original 1989 conceptualization of the World-Wide Web, and that borrows from the present Linked Data structure (cf., linkeddata.org). This higher scale network could be represented as an interconnection of texts via hyper-text links. A health information-Wayback structure, that is directly connected to, and is an integral part of the Wayback database, could be constructed, which would for instance comprise the topic osteoimmunopathology in HIV \& AIDS [9]. This novel perspective on HIT structure, which ought to be articulated in view of future improvement in the technology, such as the Semantic web meta-data edifice, would represent an ensemble of universes of data and information that could be collected, connected, and linked - from the basic fundamental osteoimmune data describing the intertwined cross-talk between cellular immunity and bone metabolism during development and aging in health, and in HIV disease \& AIDS related pathologies, to the genomic and proteomic signatures of specific osteo-immunopathologies, and could include subjects from rheumatoid arthritis to temporomandibular joint disorders, to the best available evidence for diagnosis and prognosis, critical summaries and lay-language summaries, and conceptual and textual data mining as outlined above - with patient histories, values and preferences (inter-linked at this point with other databases) in order to ensure the best available patient-centered health bioinformation technology-based clinical decisionmaking database of databases.

Wayback could sustain the expansion of health information (e.g., Health Information-Wayback; cf., Figure 1), and proffer the structure for the orderly categorization of all of the available evidence on any given condition diagnosis or prognosis, including related systematic review, and validated and quantified critical reviews and lay-language summaries. Such a cluster-randomized multi-level structure [10] would require novel complex analyses of units at each level, with more complex permutations of data for power analyses, in comparative efficacy and effectiveness research and analysis for health care practice.

Because of inherent limitations of Wayback technology, such as limited efficiency or "intelligence", the new frontier a "Semantic Web" (semanticweb.org) is presently being conceptualized that would empower searches to infer more "intelligently" what is being discussed or stored. The Semantic Web application would be more powerful than Wayback in that it could more efficiently leverage meta-data - information about information - through the standardization of meta "tags". In this manner, each web contributor will use the same syntax to describe in explicit detail what was being posted on individual site, which will allow high efficiency search engine optimization, speed and reliability. Whereas currently available search engines infer meta-data by searching and attempting to read for contextual relevance, the Semantic Web search engine will have superior rigor and robustness in looking for, and in categorizing information. The widespread use of the Semantic Web structure, when perfected, will obviate the need for systems such as Wayback to infer information cataloging, thus devoting more processing power towards organizing and presenting the information.

In brief, evidence-based standards of care pertain to crafting and implementing patient-centered treatment interventions based on the best available evidence. The quantification, analysis and dissemination of the textual content of systematic reviews and of their summaries in the form of critical reviews and lay-language summaries represent the next frontier of bioinformation for evidence-based standards of care.

\section{Acknowledgments:}

No funding or grants have been received, and no conflict of interest is present in this study. The authors thank the Evidence-Based Decisions Active Groups of Stakeholders (EBDAGS) of the EBD-Practice-Based Research Network and the EBD Study Group for the invaluable critical contributions to this work.

\section{References:}

[1] Chiappelli F \& Danaei S. Chapter 12. 2011 SpringerVerlag, Heidelberg (In Press).

[2] Ajaj RA et al. Dental Hypotheses. 2011 2:55. [doi: 10.5436]

[3] Chiappelli F \& Cajulis OS. J Evid Based Dent Pract. 2009 9:206. [PMID: 19913735]

[4] American Dental Association (2011). EBD Website, Critical Summaries, and Plain Language Summaries.

[5] Ebell MH et al. Am Fam Physician. 2004 69:548. [PMID:14971837]

[6] Bauer JG \& Chiappelli F. Open Dent J. 2010 4:133. [PMID: 20871753]

[7] Chiappelli F. Dental Hypotheses. 2010 2:105 [doi: 10.5436]

[8] Dousti M et al. Dental Hypotheses. 2010 2:165. [doi: 10.5436] 
[9] Barkhordarian A et al. Patholog Res. Int. 2011 [PMID: [10] Chiappelli F. Dental Hypotheses (In Press). 21660263]

Edited by P Kangueane Citation: Barkhordarian et al. Bioinformation 7(6): 315-319 (2011) License statement: This is an open-access article, which permits unrestricted use, distribution, and reproduction in any medium, for non-commercial purposes, provided the original author and source are credited. 


\section{Supplementary material:}

${ }^{1}$ Cf., internal document: American Dental Association: Disseminating Evidence-Based Guidelines developed by external agencies, 2011

2the level of the evidence refers to the type of study performed: clinical trial, observational study, etc. The quality of the evidence refers to how well the criteria and requirements of research methodology (i.e., sampling issues, measurement issues), of research design (e.g., sample allocation to placebo and experimental groups, drop-outs, selection of outcome variable), and data analysis (i.e., appropriate use and interpretation of statistical armamentarium) were verified in the study, regardless of its level of evidence (1). In some circles, the quality of the evidence is equated with the assessment of "risk of bias", originally proposed by the Cochrane group (Chapter 8: Assessing risk of bias in included studies. In: Higgins JPT, Green S, Eds. Cochrane Handbook for Systematic Reviews of Interventions the Cochrane Collaboration, 2011.cochrane-handbook.org). However, the "risk of bias" concept is more restrictive than the perspective of "quality of the evidence" based on the comprehensive standards of research methodology, design and data analysis. "Risk of Bias" pertain to producing a “...judgment and a support for the judgment for each entry in a 'Risk of bias' table, where each entry addresses a specific feature of the study ... assessing the risk of bias as 'low risk', as 'high risk, or as 'unclear' risk..." (note: quality assessments report a quantified value, not a subjective judgment). Furthermore, "Risk of Bias" focuses exclusively on the following potential limitations of clinical trials "...sequence generation (selection bias), allocation sequence concealment (selection bias), blinding of participants and personnel (performance bias), blinding of outcome assessment (detection bias), incomplete outcome data (attrition bias), selective outcome reporting (reporting bias) and other potential sources of bias...." (Note: quality of the evidence assessment is applicable to any and all types of research designs).

'It is important to note that the Cochrane Group proposes a similar approach, which it calls "overviews of systematic reviews", and which is discussed at length in Chap. 22 of the Cochrane Manual (Cochrane Handbook for Systematic Reviews of Interventions: Cochrane Book Series Edited by JPT Higgins \& S Green; @ 2008 The Cochrane Collaboration. ISBN: 978-0-470-69951-5.

4To be clear, the increase in memory capacity has not directly caused the increase in computational speed per se. Rather, the continual shrinking of die sizes - i.e., the physical size of the processor chip - in the manufacture of integrated circuits that has made possible this gargantuan increase in memory capacity has consequentially also led to a similar increase in computational speed. 